\title{
Les flux de traduction entre les deux rives de la Méditerranée
}

\section{Lynda Touchi-Benmansour}

\author{
CNRPAH \\ Algérie
}

Résumé : On assiste depuis quelques années à l'émergence d'une politique euroméditerranéenne visant l'échange interculturel, entre autres, par le biais de la traduction. L'analyse des flux de traduction arabe-français entre les deux rives vient conforter l'idée, déjà connue, de l'inégalité des échanges. Pour des motifs de spécialisation, nous nous limiterons, dans la présente étude, au domaine des sciences humaines et sociales avec cependant une référence aux traductions littéraires éditées au cours des deux dernières décennies à titre de comparaison.

Mot-clés : flux de traduction, arabe, français, interculturel

\begin{abstract}
The past few years have seen the emergence of a Euro-Mediterranean policy for intercultural exchange, amongst others, the translation of written literature. Flow analysis of Arabic-French translations between the two sides reinforces the idea, already known, of unequal exchange. For reasons of specialization, in this study we will limit ourselves to the translation of works from the humanities and social sciences, referring, however, to literary translations published in the past two decades, for comparison.
\end{abstract}

Keywords: translation flow, Arabic, French, intercultural

\section{Introduction}

Traduire dans le monde arabe, est avant tout une histoire d'arabisation. Ce terme est la traduction de Ta'rîb, qui signifie rendre arabe un mot ou une réalité. Dans son article intitulé « L'arabisation au Maghreb et au Machrek », Grandguillaume (1984, 1) précise, néanmoins, qu'il existe une différence entre l'arabisation au Machrek et au Maghreb. Née au cours du XIXe siècle, à l'époque de la Nahdha ${ }^{1}$, l'arabisation au Machrek aurait été motivée par le besoin d'accéder au

\footnotetext{
${ }^{1}$ Mouvement de Renaissance.
} 
modernisme, aux nouvelles techniques développées en Occident. L'assimilation de ces techniques passait nécessairement par la création de nouvelles terminologies, mais pas seulement :

L'arabisation au Machrek, c'est un processus global, qui comporte une adaptation de la langue, un mouvement de traduction, une volonté d'ouverture à des réalités nouvelles. C'est en quelque sorte le jeu normal d'une culture vivante qui s'enrichit d'un apport extérieur qu'elle va faire sien, arabiser. (Grandguillaume 1984, 1)

Née après les indépendances, l'arabisation au Maghreb est, quant à elle, motivée par le besoin de se démarquer de l'étranger, de « réintégrer à la personnalité maghrébine, un élément essentiel qui avait été oblitéré par la colonisation » (Grandguillaume 1984, 2). Malgré cette distinction entre l'arabisation au Maghreb et au Machrek, il semble qu'aujourd'hui le souci majeur du monde arabe est de s'ouvrir encore plus aux savoirs modernes tout en enrichissant la langue arabe. Prenant conscience de l'importance des enjeux culturels de la traduction, les politiques et les intellectuels arabes décident alors de renforcer le processus de modernisation culturelle initié pour certains pays du Machreq à la fin du XIX ${ }^{\mathrm{e}}$ siècle. Dans son commentaire sur « L'état de la traduction arabe des ouvrages de sciences humaines et sociales (2000 - 2009), Mohamed-Sghir Janjar, affirme que si « la généralisation du processus d'arabisation de tous les cycles de l'enseignement est une avancée en soi », elle " nécessite impérativement et parallèlement, le développement de la pratique des langues étrangères ainsi que la diversification et l'intensification du travail de traduction » (J anjar 2010, 3) .

\section{Les flux de traduction entre le français et l'arabe}

Dans un état des lieux de la traduction en Méditerranée, édité par Transeuropéennes, revue internationale de pensée critique ${ }^{2}$, et coproduit avec la Fondation Anna Lindh ${ }^{3}$ en 2010, concernant les flux de traduction arabe-français (1985-2009), on peut lire que « la France serait [...] le premier foyer de traduction de l'arabe avec quelques 1330 titres sur 10100 au total [...] Par ailleurs, trois quarts des traductions de l'arabe vers le français seraient publiés dans l'Hexagone (1728 dans le monde)» (Varlet 2010, 2).

D’autres données, que nous retrouvons dans l'Index

Translationum 4 , citent, sans détails, deux pays du Maghreb : l'Algérie et la Tunisie. " En Algérie, deux tiers des traductions concernent la littérature contemporaine algérienne arabophone (T. Ouettar, A.

\footnotetext{
${ }^{2}$ Transeuropéennes dont le siège est à Paris, France.

${ }^{3}$ Fondation Anna Lindh dont le siège est à Alexandrie, Egypte.

${ }^{4}$ Données fondées sur les enregistrements dans les services nationaux de dépôt légal ne couvrant que la période 1979-2005 et pour certains pays assez partiellement (Varlet, 2010, 2).
} 
Benhaddouga, R. Boudjedra, W. Laredj), la plupart ayant été publiés par l'Union des écrivains algériens » (Varlet 2010, 3). Néanmoins, « sur toute la période 1979-2005, le sens arabe-français ne représente que 0,9\% de l'ensemble des traductions parues en France » (Varlet 2010, 3).

Concernant le domaine des sciences humaines et sociales, qui nous intéresse particulièrement, les auteurs de cet état des lieux confirment l'extrême rareté des ouvrages traduits de l'arabe (une trentaine) sur la même période (1985-2009). Pour Varlet, « la très faible proportion des ouvrages traduits de l'arabe pourrait être mise en relation avec le statut minoritaire dans lequel est aujourd'hui tenue cette langue en France, en particulier dans l'enseignement secondaire » (Varlet 2010, 4). Dans un article intitulé « L'enseignement de l'arabe dans l'institution scolaire française », Bruno Levallois affirme que celuici « représente environ 0,17\% par rapport à l'ensemble des langues enseignées en France » (Levallois 2009, 7).

Mais, les bases de données bibliographiques (Translationum et

Electre) montrent un bond significatif de la traduction de l'arabe en

France vers les années 1990 (68 titres en moyenne par an). Selon l'analyse de Richard Jaquemond, cette augmentation tient à deux facteurs : « la hausse de la production traduite en littérature moderne entre 1991-2004 (+448\% par rapport à la décennie 1979-1990) et dans la catégorie religion (+672\%). » (2010, 362-363)

\section{Bref historique du mouvement de la traduction arabe- français}

En remontant l'itinéraire de la traduction arabe, depuis la naissance du mouvement de traduction arabe-latin à l'époque médiévale et son rôle dans le transfert de savoirs dans l'espace euroméditerranéen ${ }^{5}$, nous nous rendons compte que la contribution de la France est minoritaire comparée à celle de l'Espagne (école de Tolède) ou de l'Italie (Sicile). Comme nous l'avons déjà souligné dans notre article intitulé « La note du traducteur dans les retraductions du Coran » (Touchi-Benmansour 2012, 330) le mouvement orientaliste né à cette époque est principalement orienté vers les textes fondateurs de l'islam et le patrimoine classique arabe. C'est dans ce cadre précis que seront publiées les premières traductions françaises du Coran (1647-1990) et

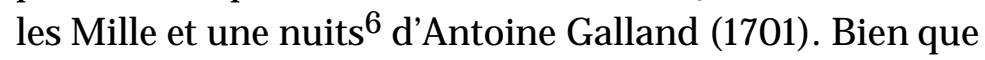

\footnotetext{
5 À ce sujet voir l'article de Daniel G. König, « Traductions et transferts de savoirs, À propos des relations entre l'Occident latin et le monde arabo-musulman», Trivium (en ligne), 8 / 2011 , consulté le 05 août 2013.

6 Contes d'origine persane, traduits en arabe à la fin du VII ${ }^{\mathrm{e}}$ siècle. Les contes des Mille et une nuits proviennent essentiellement de trois grands fonds principaux, une source indo-persane à coloration hellénistique se situant entre les $\mathrm{III}^{\mathrm{e}}$ et $\mathrm{VII} \mathrm{e}^{\mathrm{e}}$ siècle, un fonds arabe datant de la période du pouvoir des califes de Bagdad entre les IX ${ }^{\mathrm{e}}$ et XI ${ }^{\mathrm{e}}$ siècles enfin, un fonds populaire égyptien datant des $\mathrm{XII}^{\mathrm{e}}$ et XIII ${ }^{\mathrm{e}}$ siècles qui ont
} 
d'origine persane, les contes des Mille et une nuits sont souvent classés dans le patrimoine littéraire arabe.

Ce n'est que vers les années 1960 que le mouvement de traduction en France commence à se tourner vers les productions littéraires modernes avec les traductions de quelques auteurs égyptiens dont Tawfiq al-Hakim (publié aux éditions Plon), Mahmoud Teymour (aux nouvelles éditions latines) et Taha Hussein (1889-1973), sans doute le plus renommé de tous avec son autobiographie, Kitab al.Ayyam, paru chez Gallimard sous le titre de Le livre des jours ${ }^{7}$.

Il faudra attendre les années 70 et la création de la « Biliothèque arabe » en France, chez l'éditeur Jérôme Martineau, pour voir apparaître les premières traductions en sciences humaines et sociales.

\section{La nouvelle dynamique de traduction en Algérie à l'aube du XXI' siècle}

$\mathrm{Au}$ Maghreb, et plus particulièrement en Algérie, la politique d'arabisation est renforcée par la traduction vers l'arabe d'ouvrages essentiellement en anglais et en français. Depuis 2003, une série de manifestations culturelles de grande envergure contribue à cette dynamique : l'Année de l'Algérie en France en 2003, qui a favorisé la traduction vers le français, et Alger, Capitale de la Culture Arabe en 2007, qui a favoriséla traduction vers l'arabe. N'ayant pas pu aocéder à une quelconque base de données sur les traductions produites dans le cadre de la manifestation culturellel'Année de l'Algérie en France (2003), nous nous bornerons à l'examen des traductions exécutées dans le cadre de la manifestation d'Alger, Capitale de la Culture Arabe 2007.

Selon l'état de lieux édité par Transeuropéennes, cette dernière manifestation a eu pour effet principal d'intensifier l'édition et la traduction, surtout dans le domaine littéraire. Les textes choisis pour la traduction relèvent essentiellement de la littérature algérienne d'expression française. On note, cependant, une prédilection pour le texte historique : le mouvement de traduction déclenché par cette manifestation culturelle est consolidé par la création récente de l'Institut Supérieur Arabe de la Traduction ${ }^{8}$ (ISAT) qui rend possible la publication de 1000 titres (édition, réédition, traduction) par des

continué à se transformer, par suppressions ou adjonctions continues, jusqu'au XVI e siècle.

7 Traduit de l'arabe par J ean Lecerf et Gaston Wiet, paru en 1947. La dernière partie de l'autobiographie « la vie intérieure » est traduite par et publiée chez le même éditeur en 1992.

8 Institut qui active sous l'égide de la Ligue arabe. Établi à Alger en 2004, il signe en 2006 une convention de coopération avec l'université Stendhal de Grenoble (France). Cette convention porte sur l'échange d'expériences et de connaissances et la formation des étudiants en post graduation ((Magister et Doctorat) dans les domaines de l'interprétariat et de la traduction. En 2007, on lui confie la traduction de 100 ouvrages vers l'arabe. 
éditeurs privés et des institutions de recherche, dont le Centre National d'Etudes Historiques, Anthropologiques et Historiques (CNRPAH). La liste des ouvrages traduits vers l'arabe par l'ISAT, comprend une centaine de titres dont 1 en anglais, The American Captives de J.Wilson (dans la catégorie histoire) et 2 en amazigh (Amtawn Nelferh d'Abdelouahab Hamou Fekhar et Inna-yas Ccix Muhend de Mouloud Mammeri). L'édition est majoritairement partagée entre des maisons d'édition algériennes (Barzakh, El.Ikhtilef...) et françaises, avec un apport très discret du CNRPAH (5 ouvrages spécialisés). Les lacunes observées sur la liste de l'ISAT

(informations tronquées et peu systématiques, absence de catégories ou genres) rendent la classification par genre difficile. Même si la lecture et l'analyse des titres ne donne qu'une estimation approximative du domaine traduit, nous avons pu vérifier un bon nombre d'entre eux grâce à l'indexation sur réseau fournie par la bibliothèque du CNRPAH. Aussi, nous pouvons dire qu'au moins $50 \%$ des ouvrages traduits du français vers l'arabe relèvent du domaine de l'histoire (dont 1 en préhistoire) et de l'anthropologie socio-culturelle. Les ouvrages de littérature (roman, nouvelle, poésie, théâtre) représentent 1/3 de la production traduite avec une trentaine d'ouvrages. Une part insignifiante est accordée à la linguistique (1 ouvrage) et au livre pratique (2 ouvrages).

De son côté, le fonds documentaire du CNRPAH offre une liste, très réduite, d'ouvrages traduits en arabe sur l'histoire des berbères, la naissance de l'État algérien, l'art et la poésie populaire algérienne, les mosquées de la ville d'Alger, la préhistoire en Algérie et l'anthropologie socio-culturelle ${ }^{9}$. Ces deux dernières thématiques ont fait l'objet de trois traductions ${ }^{10}$ éditées par ledit centre de recherche. Bien entendu, celles-ci sont loin d'être suffisamment représentatives pour faire partie de l'état des lieux, mais méritent quand même d'être citées parmi les efforts de traduction.

Cette dynamique créée par les manifestations culturelles se poursuit après 2007. Voici à titre indicatif, les titres traduits aux éditions Barzakh, spécialisées en littérature, sur la période 2001-2013, et ceux traduits, durant la même période, aux éditions Al. Ikhtilef : 
Tableau 1 (Établi suivant une liste fournie par les Éditions Barzakh, Alger)

\begin{tabular}{|c|c|c|c|c|}
\hline $\begin{array}{l}\text { Ouvrages } \\
\text { traduits vers le } \\
\text { français }\end{array}$ & $\begin{array}{l}\text { Titre } \\
\text { original en } \\
\text { arabe }\end{array}$ & Auteurs & Traducteurs & $\begin{array}{l}\text { Catégorie, } \\
\text { Année }\end{array}$ \\
\hline $\begin{array}{ll}\text { 1- } & \text { Sebeiba- } \\
& \text { Tillellin }\end{array}$ & - & M.Bouzid & $\begin{array}{l}\text { L.Touchi - } \\
\text { Benmansour }\end{array}$ & $\begin{array}{l}\text { Anthropologie } \\
2001\end{array}$ \\
\hline 2- Zana & & H. Ayachi & Y. Mila & $\begin{array}{l}\text { roman, 2003- } \\
2004\end{array}$ \\
\hline $\begin{array}{l}\text { 3- Les Voies de } \\
\text { l'errance }\end{array}$ & & A. Benmansour & L.Nia & roman, 2012 \\
\hline $\begin{array}{l}\text { 4- Journal d'une } \\
\text { femme } \\
\text { insomniaque }\end{array}$ & & $\begin{array}{l}\text { Rachid } \\
\text { Boudjedra }\end{array}$ & A.Moussali & roman, 2012 \\
\hline 5- Labyrinthes & & H.Ayyachi & L.Nia & $\begin{array}{l}\text { roman, en } \\
\text { cours }\end{array}$ \\
\hline
\end{tabular}

Tableau 2 (Établi suivant une liste fournie par les Éditions Barzakh, Alger)

\begin{tabular}{|c|c|c|c|c|}
\hline $\begin{array}{l}\text { Ouvrages } \\
\text { traduits vers } \\
\text { l'arabe }\end{array}$ & $\begin{array}{l}\text { Titre original } \\
\text { en français }\end{array}$ & Auteurs & Traducteurs & $\begin{array}{l}\text { Catégorie, } \\
\text { Année }\end{array}$ \\
\hline & L'Aube Ismaël & M.Dib & M.Hakim & $\begin{array}{l}\text { roman, 2003- } \\
2004\end{array}$ \\
\hline & L'écrivain & Y.Khadra & I.Bioud & roman, 2007 \\
\hline & $\begin{array}{l}\text { Cinq } \\
\text { fragments du } \\
\text { désert }\end{array}$ & R.Boudjedra & M.Hakim & roman, 2007 \\
\hline & $\begin{array}{l}\text { La vieà } \\
\text { l'endroit }\end{array}$ & R.Boudjedra & A.Meziane & roman, 2012 \\
\hline & $\begin{array}{l}\text { La martingale } \\
\text { algérienne }\end{array}$ & A.Hadj-Nacer & A.B Bakeli & roman, 2007 \\
\hline & Tuez-les tous & S.Bachi & M.Sari & roman, 2008 \\
\hline & Le petit prince & $\begin{array}{l}\text { A.D Saint- } \\
\text { Exupéry }\end{array}$ & $\begin{array}{l}\text { L.Brousse, } \\
\text { Z.Talbi }\end{array}$ & roman \\
\hline & Ali Boumendjel & M.Rahal & F.Guechi & $\begin{array}{l}\text { roman, en } \\
\text { cours }\end{array}$ \\
\hline & $\begin{array}{l}\text { Le choix de } \\
\text { l'Algérie }\end{array}$ & P. et C. Chaulet & Z.Koubi & $\begin{array}{l}\text { roman, en } \\
\text { cours }\end{array}$ \\
\hline & $\begin{array}{l}\text { Hegel : } \\
\text { l'inquiétude du } \\
\text { négatif }\end{array}$ & J ean-Luc Nancy & $\begin{array}{l}\text { B.M } \\
\text { Bencharki }\end{array}$ & $\begin{array}{l}\text { Philosophie, } \\
2010\end{array}$ \\
\hline
\end{tabular}


Tableau 3 (Établi suivant une liste fournie par les Éditions Al. Ikhtilef, Alger)

\begin{tabular}{|c|c|c|c|c|}
\hline $\begin{array}{l}\text { Ouvrages } \\
\text { traduits vers } \\
\text { l'arabe }\end{array}$ & $\begin{array}{l}\text { Titre original } \\
\text { en français }\end{array}$ & Auteurs & Traducteurs & $\begin{array}{l}\text { Catégorie, } \\
\text { Année }\end{array}$ \\
\hline & $\begin{array}{l}\text { L'invention du } \\
\text { quotidien } \\
\text { La philosophie } \\
\text { herméneutique } \\
\text { L'Éthique de la } \\
\text { discussion } \\
\text { Le } \\
\text { monolinguisme } \\
\text { del'autre } \\
\text { Je m'appelle } \\
\text { François } \\
\text { L'esthétique } \\
\text { contemporaine } \\
\text { Capitalisme ou } \\
\text { Démocratie? } \\
\text { Les Politiques } \\
\text { linguistiques } \\
\text { ? } \\
\text { ? } \\
\text { La langue, } \\
\text { l'imaginaire et le } \\
\text { symbolique } \\
\text { Les termes clés de } \\
\text { l'analyse de } \\
\text { discours } \\
\text { Le tournant } \\
\text { herméneutique de } \\
\text { la } \\
\text { phénoménologie } \\
\text { Le Libraire } \\
\text { Kaboul } \\
\text { Réflexion faite }\end{array}$ & $\begin{array}{l}\text { M. De Certeau } \\
\text { H.G. Gadamer } \\
\text { J. Habermas } \\
\text { J. Derrida } \\
\text { C.Dantzig } \\
\text { M.J imenez } \\
\text { M.Fleurbaey } \\
\text { L-J Calvet } \\
\text { ? } \\
\text { ? } \\
\text { J.Lacan } \\
\text { P.Ricoeur } \\
\text { D. Seierstad } \\
\text { Maingueneau } \\
\text { J. Grondin }\end{array}$ & $\begin{array}{l}\text { M.C. Ezzine } \\
\text { M.C. Ezzine } \\
\text { O.Mhibel } \\
\text { O.Mhibel } \\
\text { A.El.Mawla } \\
\text { K.Boumnir } \\
\text { M.Yahyaten } \\
\text { ? } \\
\text { A.Doudou } \\
\text { H.Nasr } \\
\text { O.Mhibel } \\
\text { M.Yahyaten }\end{array}$ & $\begin{array}{l}\text { Philosophie, } \\
2011 \\
\text { Philosophie, } \\
2002 \\
\text { Philosophie, } \\
2009 \\
\text { Philosophie, } \\
2007 \\
\text { roman, 2008 } \\
\text { Philosophie, } \\
2012 \\
\text { Philosophie, } \\
2007 \\
\text { Langue, } 2008 \\
\text { roman, 2008 } \\
\text { roman, 2003 } \\
\text { Philosophie, } \\
2006 \\
\text { Littérature, } \\
2005 \\
\text { Philosophie, } \\
2006 \\
\text { Autobiogra- } \\
\text { phie 2006 }\end{array}$ \\
\hline
\end{tabular}

Malgré les lacunes (absence du titre original, manques dans la case auteurs ...) observées dans la liste fournie par ce dernier éditeur, la case « catégorie » nous permet d'avancer, avec une certaine réserve, qu'il existe une réelle tendance de la traduction à partir de 2003 vers les ouvrages de pensée et de philosophie française et allemande : sur 30 ouvrages traduits aux éditions Al.Ikhtilef, on compte une quinzaine spécialisés en philosophie, soit 50\%, une douzaine en littérature 
(roman) et une part insignifiante en linguistique (1 ouvrage), avec une orientation unique français-arabe. La tendance est inversée aux éditions Barzakh : 99\% des traductions sont des romans. Là aussi le sens français-arabe est privilégié, même si la traduction vers le français représente 1/3 de l'ensemble des traductions, ce qui mérite d'être souligné. Si nous avons choisi de mentionner ces deux éditeurs, ce n'est pas par hasard : le choix d'une politique de partenariat avec des

éditeurs arabe et français (Actes Sud, ), connus sur le marché international du livre, leur a permis de rentrer dans une dynamique évolutive réelle en matière de traduction.

\section{L'édition des traductions arabes d'ouvrages en sciences humaines et sociales}

La traduction des sciences humaines et sociales, dont l'anthropologie socio-culturelle et la préhistoire, n'a pas encore reçu l'attention qu'elle mérite, ni de la part des centres de recherches où elle s'exerce de manière sporadique et ponctuelle, ni de la part des chercheurs dont l'unique souci est de pouvoir publier en anglais pour être répertoriés dans les réseaux internationaux de la recherche scientifique. La question ne reçoit pas plus d'égard de la part des

éditeurs qui concentrent leurs productions sur la littérature (poésie, romans de jeunesse, beaux livres). Concernant l'édition au Maghreb, et s'appuyant sur les données de l'Index translationum, Varlet estime qu'« En Algérie, les deux tiers des traductions concernent la littérature contemporaine arabophone » (3). Ces données sont comblées par les travaux ultérieurs de Hasnaa Dessa et Mohamed-Sghir Janjar menés dans le cadre du même programme « Traduire en Méditerranée » en collaboration avec la Fondation du Roi Abdul-Aziz pour les études islamiques et sciences humaines à Casablanca. Selon Hasnaa Dessa et Mohamed-Sghir J anjar, ces estimations doivent être relativisées à cause des déficits de la base de données translationum : " Les travaux de Richard J aquemond ont montré le caractère parcellaire des données collectées par les sources bibliographiques internationales comme l'Index Translationum développé par l'Unesco, en matière d'édition et de traduction arabes » (Dessa, J anjar 2010, 5). Les travaux de Hasnaa Dessa et Mohamed-Sghir J anjar soulignent au passage « les décalages énormes qui existent entre les informations qu'offrent la banque de données de la Fondation et celles que présentent de telles sources » (Dessa, Janjar 2010, 5). Ils concernent l'état de la traduction des ouvrages en sciences humaines et sociales au cours de la dernière décennie. Le fonds de traduction, dont la mise à jour s'est poursuivie jusqu'en 2010, atteint le seuil de 11.000 ouvrages traduits en arabe. Les auteurs de l'état des lieux signalent une croissance significative dans la production des traductions par rapport à la décennie précédente (Dessa, J anjar 2010, 6) et une évolution inégale selon les pays. 
En dehors des centres de recherches spécialisés, la publication des traductions arabes d'ouvrages en sciences humaines et sociales est prise en charge par les éditeurs des secteurs public et privé, avec la nette suprématie de ce dernier dans la majorité des pays arabes ${ }^{11}$. Pourtant, les chiffres fournis par la Fondation du Roi Abdul-Aziz pour les Études Islamiques et les Sciences Humaines confirment la rareté des productions dans ce domaine due au coût élevé de la traduction.

\section{Les langues-source, auteurs et champs disciplinaires les plus traduits}

\section{Les langues-source}

Parmi les langues-source ${ }^{12}$ des traductions arabes en sciences humaines et sociales, le français occupe la seconde place après l'anglais. $\mathrm{Au}$ Maghreb, la majorité des traducteurs « utilise les sources françaises aussi bien pour traduire les auteurs français que les auteurs nonfrançais » (Dessa, Janjar 2010, 13) ; il s'agit bien de traductions relai ou retraductions ${ }^{13}$. On note également " l'essor relatif des traductions arabes des productions intellectuelles iraniennes [...], le peu d'intérêt accordé à l'espagnol, l'allemand, le russe et aux langues de l'extrêmeOrient [...] et l'absence de traductions arabes à partir des langues anciennes » (Dessa, J anjar 2010, 13-14).

\section{Les auteurs}

Parmi les auteurs les plus traduits au Maghreb sont cités : Paul Ricoeur, Pierre Bourdieu, J aques Derrida, Robert Blanchet, Tzvetan Todorov ${ }^{14}$, Malek Bennabi, Mohamed Arkoun pour le français, Noam Chomsky, Edward Said, Bernard Lewis, Bertrand Russell, Anthony

Giddens pour l'anglais, Friedrich Nietzche, Jürgen Habermas pour l'allemand, Umberto Eco pour l'italien, AbdolKarim Sourouch pour le persan et Said Nawrassi pour le turc. On constate que la grande majorité des traductions concerne la production intellectuelle française et la production d'auteurs arabes d'expression française (Malek Bennabi, Mohamed Arkoun, Edward Said).

Dans le sens inverse, les auteurs arabes contemporains les plus traduits sont : le philosophe syrien Sadiq Jala Al-Azm (traduit récemment en français), Edward Said et Nawal Al-Sadawi (traduits dans les langues européennes et en turc à partir de l'anglais), Mohamed Arkoun, Fatima Mernissi, Tariq Ramadan, Moustapha Safouan (traduits dans les langues européennes et en turc à partir du français).

\footnotetext{
11 À l'exception de l'Egypte et des pays du Golfe où l'on note le quasi monopole du secteur public.

12 Principalement l'anglais, le français, le persan, l'allemand, l'espagnol, le russe, l'hébreu.

13 Les textes de Nietzche, Eco et Habermas publiés au Maghreb sont traduits à partir des éditions françaises.

14 On retrouve dans une plus faible proportion les travaux de Gilles Deuleuze, Michel Foucault, J ean Baudrillard, Edgar Morin, Marcel Gauchet, René Girard.
} 
Ce nombre extrêmement réduit d'auteurs ne reflète pas la réalité des productions arabes dans un domaine aussi riche que celui des sciences humaines et sociales.

On note, par ailleurs, la traduction vers le français et l'anglais de quelques rares penseurs arabes classiques : plus de 310 titres en religion entre 1985-2009. En philosophie classique, sont cités « AlGhazali, le plus traduit de tous avec 26 titres chez les plus grands éditeurs arabes et français, Ibn Arabi avec 22 titres et la même diversité de structures éditoriales [...] Ibn Rushd, Al-Farabi, Ibn Sina » (Varlet 2010, 10-11). Nous pouvons rajouter à cette liste Ibn Tufail avec son roman philosophique Hayy Ben Yaqdhân, Ibn Battuta avec sa Rihla, Al-Idrissi avec sa Nuzhat al.Mushtaq (également connue sous Le livre de Roger) et J ean Léon l'Africain avec Description de l'Afrique.

Les champs disciplinaires

Selon le classement Dewey (15-16), réalisé par les services documentaires de la Fondation, les six premières disciplines les plus traduites dans le monde arabe sont : la socio-anthropologie, l'Histoire, les sciences politiques, les études littéraires et linguistiques, la philosophie et les études islamiques.

\section{L'inégalité des échanges entre les deux rives}

Dans ses conclusions générales et recommandations, l'état des lieux de la traduction dans la région euro-méditerranéenne « révèle un déficit quantitatif et qualitatif général de traduction et une inégalité flagrante entre les deux rives. Il est une plongée dans la double réalité des logiques de centralités et de périphéries qui structurent les échanges dans la région et des hégémonies culturelles qui y prévalent» (Deschaumes 2010, 13). L'analyse des chiffres fournis par les sources bibliographiques et les enquêtes de terrain amène les auteurs de l'état des lieux aux conclusions suivantes :

a) dans la majorité des pays de l'Union européenne, on traduit un livre de l'arabe pour mille traductions ;

b) l'arabe comme langue source ne représente que 0,64\% des œuvres traduites en français entre 1985-2000 ;

c) les pays qui ont traduit le plus de l'arabe dans les 20 à 25 dernières années sont la Turquie (1161) et la France (1065) ${ }^{15}$ Les traductions portent essentiellement sur la littérature arabe moderne d'auteurs égyptiens (dont Taha

Hussein, Sahar Khalifa, Naguib Mahfouz, Sonnallah Ibrahim, Gamal Ghithany, Alaa El-Aswany), palestiniens (Mahmoud Darwich), algériens (Rachid Boudjedra, Waciny

15 La Bosnie Herzégovine (513), l’Allemagne et la Suisse (508), l’Espagne (472 sur la période 1995-2010), l'Italie (317), la Serbie (147). Les pays ayant traduit entre 50 et 60 titres sur la même période sont la Slovénie, la Roumanie, la Pologne et la Bulgarie. 
Laaredj, Ahlam Mostaghenmi) et libanais (Hanane

El.Cheikh, Elias Khoury, Leila Baalabaki, Nadjwa Barakat).

Le peu de traductions qui existent (une trentaine en 25 ans en Français, par exemple) portent, selon Richard Jacquemond ${ }^{16}$, sur " deux domaines de prédilection : les débats et polémiques autour de la place de l'Islam dans les sociétés arabes modernes [...], et ceux autour de la question féminine ».

Toujours selon J acquemond, « l'étude comparée des flux de traduction du français vers l'arabe et, à l'inverse, de l'arabe vers le français, offre une illustration assez exemplaire de l'échange culturel inégal entre une langue centrale ou dominante (le français) et une langue périphérique ou dominée (l’arabe) » (J acquemond 2008, 347). En ce qui nous concerne, cette vision tout à fait subjective et unilatérale des langues orientales ou chamito-sémitiques comme l'arabe, ne peut qu'entraver l'échange entre les cultures. Des langues comme l'arabe et le chinois présentent un paradoxe évident : langues de civilisations anciennes, langues internationales avec plus de 250 millions de locuteurs, langues officielles et de travail à l'ONU, elles ne représentent pourtant que $1 \%$ du marché de la traduction et occupent donc une « place périphérique » selon la définition d’Heilbron $(1999,434)$.

En réalité, la question de l'inégalité des échanges en termes de traduction entre les deux rives tient de plusieurs facteurs : pour Ghislaine Glasson Deschaumes « elle procède souvent dans le monde arabe d'un autodénigrement, et, en Europe, d'une attitude de mépris » (État des Lieux 36), alors que, pour Mohamed-Sghir Janjar, la question est plus complexe : selon lui, « les travaux arabes pour lesquels il est difficile de trouver traducteurs ou réception en Europe sont ceux qui n'utilisent pas les codes, les outils et les réflexes ancrés dans le champ académique européen depuis plus d'un siècle ». » (in Deschaumes 2010, 36)

\section{Conclusion}

La traduction en arabe est en nette augmentation depuis la dernière décennie et dans tous les domaines. Cela est dû, en partie, à « l'essor de l'édition privée dans les pays arabes ». L'intensification du processus de traduction dans le monde arabe (Moyen Orient, pays du Golfe et Maghreb), au cours des deux dernières décennies, confirme la volonté des politiques et intellectuels arabes de s'ouvrir aux savoirs modernes de l'Occident, comme ce le fut à l'époque classique avec les savoirs venus de la Grèce, de l'Inde et de la Chine. Le domaine de la traduction en sciences humaines et sociales est, néanmoins, le plus inégalitaire en termes d'échange interculturel entre les deux rives de la Méditerranée. Car, si le volume des ouvrages traduits en arabe dans le

16 Cité par Ghislaine Glasson Deschaumes (2010, 35). 
domaine des sciences humaines et sociales demeure insuffisant, que dire de la traduction des productions arabes vers les langues de l'Union européenne, le turc et l'hébreu?

\section{Références bibliographiques}

Deschaumes, Ghislaine. État des lieux de la traduction dans la région euroméditerranéenne. Alexandrie : Transeuropéenne, Paris \& Fondation Anna Lindh, 2010.

Dessa, Hasnaa. État de la traduction arabe des ouvrages de sciences humaines et sociales (2000-2009). Alexandrie : Transeuropéenne, Paris \& Fondation Anna Lindh, 2010.

J anjar, Mohamed-Sghir. État de la traduction arabe des ouvrages de sciences humaines et sociales (2000-2009). Alexandrie : Transeuropéenne, Paris \& Fondation Anna Lindh, 2010.

Grandguillaume, Gilbert. Les relations entre le Maghreb et le Machrek. Des solidarités anciennes aux réalités nouvelles. Cahiers du GIS « sciences humaines sur l'aire méditerranéenne », Cahier $\mathrm{N}^{\circ}$ 6, CNRS, Institut de Recherches Méditerranéennes, Université de Provence, 1984.

Jacquemond, Richard. "Les flux de traduction entre le français et l'arabe depuis les années 1980 : un reflet des relations culturelles ». In : Gisèle Sapiro (dir.), Translatio. Le marché de la traduction en France à l'heure de la mondialisation. Paris : CNRS Editions, 2008 : 347-369.

König, Daniel G. « Traductions et transferts de savoirs, À propos des relations entre l'Occident latin et le monde arabo-musulman». Trivium (en ligne), 8 /2011. URL: http:// trivium.revues.org/ (Consultéle 05 août 2013).

Levallois, Bruno. "L'enseignement de l'arabe dans l'institution scolaire française ». In : Langues et Cité, n 15,2009, p. 7.

Varlet, Emmanuel. Etude sur les flux de traduction arabe français (19852009). Alexandrie : Transeuropéenne, Paris \& Fondation Anna Lindh, 2010. 